\title{
Association between lymphocyte expression of the apoptotic receptor Fas and pain in critically ill patients
}

\author{
Elizabeth DE \\ Papathanassoglou',* \\ Meropi DA Mpouzika²,* \\ Margarita Giannakopoulou ${ }^{3}$ \\ Evangelos Bozas ${ }^{3}$ \\ Nicos Middleton ${ }^{2}$ \\ George Tsiaousis ${ }^{2}$ \\ Andreas Karabinis s,5 $^{4}$
}

'Faculty of Nursing, University of Alberta, Edmonton, AB, Canada; ${ }^{2}$ Department of Nursing, Cyprus University of Technology, Limassol, Cyprus; ${ }^{3}$ Department of Nursing, School of Health Sciences, National and Kapodistrian University of Athens, ${ }^{4}$ Surgical Care Unit, The Onassis Cardiac Surgery Center, Kallithea, ${ }^{5}$ School of Medicine, National and Kapodistrian University of Athens, Athens, Greece

*These authors contributed equally to this work
Correspondence: Elizabeth DE Papathanassoglou

Faculty of Nursing, University of Alberta, Edmonton Clinic Health Academy,

I | 405-87th Avenue, Edmonton, AB T6G

IC9, Canada

Tel +l 7804925674

Email papathan@ualberta.ca
This article was published in the following Dove Press journal:

Journal of Pain Research

13 January 2017

Number of times this article has been viewed

Objective: Lymphocyte apoptosis in critical illness is associated with immunosuppression. We explored for the first time the associations between pain ratings and expression of the apoptotic receptor Fas on $\mathrm{B}$ and $\mathrm{T}$ cells in critically ill patients and the potential mediating effects of adrenocorticotropic hormone (ACTH), cortisol, and substance P (SP).

Design: This is an exploratory correlational study with repeated measurements (14 days followup) and cross-sectional comparisons.

Setting: This study was conducted in a state hospital in the metropolitan area of Athens, Greece. Participants: The participants were 36 consecutive critically ill patients and 36 matched controls.

Outcome measures: Pain measured by the self-reported numeric rating scale [NRS], the behavioral pain scale, and the pain assessment scale was the primary outcome measure. Flow cytometry (Fas), electrochemiluminescence (ACTH and cortisol) and enzyme-linked immunosorbent assay (SP) were used. Mixed linear models for repeated measurements and bivariable associations at discrete time points were employed.

Results: Significant pain at rest was noted. Pain ratings associated with Fas expression on cytotoxic T cells $(P=0.041)$ and B cells $(P=0.005)$, even after adjustment for a number of clinical treatment factors ( $P=0.006$ and $P=0.052$, respectively). On the day that more patients were able to communicate, Fas on B cells $(r=0.897, P=0.029)$ and cytotoxic T cells $(r=0.832$; $P=0.037)$ associated with NRS ratings. Associations between pain ratings and ACTH serum levels were noted $(P<0.05)$. When stress neuropeptide levels were added to the model, the statistical significance of the associations between pain ratings and Fas expression was attenuated $(P=0.052-0.063)$, suggesting that stress neuropeptides may partially mediate the association.

Conclusion: Preliminary evidence for the association between pain and lymphocyte apoptotic susceptibility is provided. The role of pain management in maintaining immunocompetence in critical illness is worth exploring.

Keywords: lymphocyte apoptosis, pain, critical illness, adrenocorticotropic hormone, cortisol, substance $\mathrm{P}$

\section{Introduction}

Pain is a major stressor and one of the most prevalent symptoms throughout the critical illness trajectory. ${ }^{1,2}$ The majority of intensive care unit (ICU) patients report pain, while approximately 4 of every 6 critically ill patients experience moderate-to-severe pain. ${ }^{3}$ Apart from pain attributed to conditions related to admission diagnoses, care procedures are also associated with significant increases in pain. ${ }^{4}$ 
Underdiagnosed pain and unrelieved pain are associated with compromised ICU patients' outcomes, including increased dependency on mechanical ventilation, nosocomial infections, hemodynamic instability and delirium..$^{5-7}$ Pain can also compromise immune responses. In animal models, acute pain causes suppression of splenic natural killer (NK) cell activity, ${ }^{8}$ whereas in humans, adequate postoperative pain control inhibits the expected depression of lymphoproliferation ${ }^{9}$ and the suppression of NK activity. ${ }^{6}, 10$ Compromised immunity is a pivotal derangement in critical illness, associated with increased incidence and severity of infections, ${ }^{11}$ as well as with increased morbidity and mortality. ${ }^{11}$ Lymphocyte apoptosis contributes to immunosuppression in critical illness. ${ }^{12,13}$ The Fas/Fas ligand system is a receptor-ligand system involved in apoptotic signal transduction. Fas is a membrane-bound surface receptor that is involved in the control of the immune system. Expression of Fas is a measure of susceptibility to apoptosis. ${ }^{14}$ Increased lymphocytic expression of the apoptotic receptor Fas is observed in critically ill individuals and is negatively associated with lymphocytic survival. ${ }^{15}$

Research on the physiological effects of ICU pain is very scarce. Moreover, the potential involvement of pain in critical illness-related immunosuppression has not been investigated. Pain activates the stress response. ${ }^{16}$ Previous results have shown associations between stress neuropeptide levels and lymphocyte counts in critically ill individuals. ${ }^{13}$ However, the association between the intensity of pain experienced by critically ill individuals and immune outcomes has not been addressed. In this study, through a repeated measures correlational design, we aimed to explore the associations between the intensity of pain - as either reported by conscious critically ill patients or objectively assessed using behavioral pain scales - and the expression of Fas on B cells and T ( $\mathrm{T}$ helper and cytotoxic T) cells as a measure of apoptotic susceptibility. Moreover, we explored the potential mediating effects of classical stress hormones (adrenocorticotropic hormone $[\mathrm{ACTH}]$ and cortisol) and substance $\mathrm{P}(\mathrm{SP})$, which is a neurotransmitter involved in both nociception and stress perception.

\section{Methods}

\section{Research design}

This was a pilot repeated measures correlational design with cross-sectional comparisons.

This was part of a study on the role of pain and stress neuropeptides in critical illness, preliminary results of which have already been reported. ${ }^{13}$

\section{Sample and setting}

The study was conducted in the general ICU of a tertiary state hospital in the metropolitan area of Athens, Greece. Ethical approval was acquired by the hospital's ethics committee and the Department of Nursing of the National and Kapodistrian University of Athens. A written informed consent was obtained from patients or their legal representatives.

Due to the lack of previous data on the association between Fas and pain in humans on which to base power analysis, we assumed that a large effect size would be clinically meaningful. Therefore, based on a large effect size, an $85 \%$ desired power, 2 -tailed tests, and $\alpha=0.05$, the desired sample size was calculated as 35 participants.

Exclusion criteria included patients with sepsis, shock, and delirium or patients receiving corticosteroids. A blood sample was acquired through an arterial catheter every 48 hours (at $\sim 9$ am) for up to 14 days for the isolation of peripheral blood mononuclear cells (PBMC).

\section{Pain assessment}

Pain assessments were performed every 48 hours for up to 14 days. Self-reporting by conscious patients is the optimal way to rate pain in the ICU $;{ }^{17}$ however, most critically ill patients are unable to communicate. Therefore, behavioral pain rating scales for uncommunicative patients have been developed. ${ }^{18}$ The following 3 scales were employed: 1) the self-reported numeric rating scale (self-NRS; ranging from 0 , no pain, to 10 , worst imaginable pain), which could only be applied in patients who could communicate verbally or nonverbally (by pointing their response on a laminated large-print scale); 2) the pain assessment and intervention notation algorithm (pain assessment scale [PAS]) developed by Puntillo et a $1^{19}$ (scale range: 1-14), which includes several behavioral and physiological indicators organized into 4 groups (movements, facial indicators, posturing, and facial indicators) $;{ }^{19}$ and 3) the Behavioral Pain Scale (BPS) developed by Payen et $\mathrm{al}^{20}$ for sedated, critically ill patients (scale range: 3-12), which consists of a sum of scores based on facial expression, upper limb movement and compliance with ventilation. ${ }^{20}$ Non-self-report pain scales provide only indications of the presence of pain. Higher levels of pain indicators have been associated with higher pain intensity; hence, although they do not assess pain intensity per se, they are considered as the best available approximation. Herein, we used the more inclusive term "pain rating".

All scales described earlier were completed by the same specially trained nurse-investigator. Behavioral pain ratings preceded self-reported NRS ratings. All pain ratings were 
performed at rest, either before or 30 minutes after any potentially painful procedures.

\section{Clinical assessment}

Clinical severity of patients was assessed by the multiorgan failure (MOF), ${ }^{21}$ the Multiple Organ Dysfunction Score (MODS), ${ }^{22}$ and the Acute Physiology and Chronic Health Evaluation II (APACHE II) system. ${ }^{23}$

\section{Quantification of Fas expression on lymphocytes and stress neuropeptide serum levels}

Peptide levels were measured as described previously. ${ }^{13}$ In brief, cortisol and ACTH serum levels were quantified by an electrochemiluminescence immunoassay (ECLIA; Elecsys 2010 analyzer; Roche, Bohemia, NY, USA). SP was quantified by an enzyme-linked immunosorbent assay (ELISA) (Assay Designs Inc, Ann Arbor, MI, USA). Assays were run in duplicate at the same assay run. The intraassay coefficients of variation were $<5 \%$.

\section{Statistical analysis}

Mean \pm standard deviation of variable values are reported. Logarithmic transformation was performed when variables departed significantly from normality. To explore the associations between pain and Fas expression levels accounting for repeated measurements, linear mixed modeling (LMM) was employed with and without adjustment for the presence of trauma, MODS severity and administration of opioid analgesia and sedation. Additionally, neuropeptide levels were added to the model in order to explore the mediating effects. The intraclass correlation coefficient (ICC) (defined as the ratio of the between-patient to total variance) was used to quantify the amount of clustering. LMM was selected since it allows for time-varying covariates, the intrasubject correlation of response measurements and the loss of participant follow-up due to ICU discharge or transfer. We utilized the Akaike information criteria (AIC) to select for the best model fit. To confirm the repeated measures analysis at discrete time points, bivariable associations between pain ratings, serum stress neuropeptides and lymphocyte counts positive for Fas were examined at the following time points: 1) the first day in the study, 2) the day of maximum MOF severity, and 3) the day of minimum MOF severity. A nominal significance level $(\alpha=0.05)$ was used. Statistical analyses were performed using the Statistical Package for Social Sciences (SPSS Version 21).

\section{Results}

Demographics, clinical outcomes, and pain ratings

Thirty-six consecutive adult critically ill patients were enrolled in the study. All patients were Caucasian, predominantly males $(75 \%)$ and aged $51.55 \pm 18.85$ years. All patients remained nonseptic, and the mortality rate in the 14-day period of the study was $19 \%$. Control subjects had an average age of $52.36 \pm 17.63$ years. In terms of analgesia, patients received either fentanyl intravenous (IV) infusion or remifentanil IV infusion, and they received either midazolam or propofol IV for sedation. Clinical and demographic data of patients are listed in Table 1.

A total of 327 pain ratings were performed throughout the study (36 participants, 3 scales and up to 7 ratings per participant). The self-reported NRS was the least used scale: only 32 assessments were completed, due to communication limitations related to the presence of endotracheal tube, compromised neurological status and frailty. Table 1 depicts the time trend of mean values of all pain scales, through the 4 predefined time points (study entry, maximum severity day, minimum severity day, and the last day in the study). It can be noted that all ratings start off close to the median value, where they persist through the day of maximum severity, and then fall to lower scores toward the end of the study.

\section{Associations between Fas expression and pain ratings}

As reported previously, ${ }^{13}$ the expression of Fas on $\mathrm{T}$ (both helper and cytotoxic) cells and B cells was significantly higher in patients than in controls throughout the study. Bivariable linear mixed models were employed to explore the associations between pain ratings and Fas expression accounting for repeated measurements. PAS and BPS pain ratings exhibited positive associations with Fas expression on B and cytotoxic $\mathrm{T}$ cells (Table 2A). Furthermore, when multivariable mixed models were used to adjust for the presence of trauma, MODS severity and the dose of opioid analgesia and sedation, the association between pain ratings and Fas expression on cytotoxic $\mathrm{T}$ cells remained significant; however, the association with Fas expression on B cells exhibited a nonsignificant trend for positive association (Table 2B). No significant associations were noted with regard to Fas expression on $T$ helper cells.

These associations were confirmed when bivariate crosssectional associations were explored. No significant associations were noted with regard to Fas expression on helper T cells (Table 3 ). 
Table I Clinical and demographic data of patients

\begin{tabular}{|c|c|c|c|c|}
\hline \multicolumn{5}{|c|}{ A. Demographic and admission data } \\
\hline Characteristic & & & $\%(n)$ & Mean \pm SD \\
\hline \multicolumn{5}{|l|}{ Gender } \\
\hline Male & & & $75 \%(27)$ & \\
\hline Female & & & $25 \%(9)$ & \\
\hline Age & & & & $51.55 \pm 18.85$ \\
\hline \multicolumn{5}{|l|}{ Admission diagnosis } \\
\hline Head injury & & & $25 \%(9)$ & \\
\hline Multiple trauma (nonneuro) & & & $16.7 \%(6)$ & \\
\hline Cerebral hemorrhage & & & $16.7 \%(6)$ & \\
\hline Surgical/postoperative complications & & & $13.8 \%(5)$ & \\
\hline Medical/metabolic & & & $2.7 \%(I)$ & \\
\hline Respiratory & & & $8.3 \%(3)$ & \\
\hline Cardiac & & & $5.6 \%(2)$ & \\
\hline Neurologic/neurosurgery & & & $5.6 \%(2)$ & \\
\hline Vascular disorders/embolism & & & $5.6 \%(2)$ & \\
\hline \multicolumn{5}{|l|}{ B. Clinical data } \\
\hline Scale (scale range) & First day & Day of maximum severity & Day of minimum severity & Last day \\
\hline MOF (0-14) & $5.40 \pm 0.89$ & $5.73 \pm 1.12$ & $4.74 \pm I .75$ & $4.58 \pm 1.97$ \\
\hline MODS (0-24) & $4.40 \pm 2.30$ & $6.33 \pm 2.86$ & $5.48 \pm 2.54$ & $5.88 \pm 5.5$ \\
\hline APACHE II (0-7I) & $|4.80 \pm 5.7|$ & $20.3 \pm 7.26$ & $19.67 \pm 5.99$ & $19.13 \pm 7.43$ \\
\hline Pain NRS self-assessment $(0-10)$ & $4.20 \pm 2.86$ & $4.37 \pm 2.56$ & $3.28 \pm 2.28$ & $2.92 \pm 2.29$ \\
\hline PAS $(I-14)$ & $5.80 \pm 3.63$ & $5.93 \pm 3.03$ & $5.00 \pm 3.05$ & $3.92 \pm 3.42$ \\
\hline BPS (3-12) & $6.00 \pm 2.82$ & $6.15 \pm 2.77$ & $4.7 I \pm I .38$ & $4.72 \pm 1.25$ \\
\hline
\end{tabular}

Notes: (A) Demographic data and admission diagnoses. (B) Clinical data (mean \pm SD) of study subjects at the first and last days of the study and days of maximum and minimum severity (based on MOF scores).

Abbreviations: APACHE II, Acute Physiology and Chronic Health Evaluation II; BPS, behavioral pain scale; MODS, Multiple Organ Dysfunction Score; MOF, multiorgan failure; NRS, numeric rating scale; PAS, pain assessment scale; SD, standard deviation.

\section{Associations between stress neuropeptide levels and pain ratings}

To investigate the potential mediating effects of stress neuropeptides in the association between pain ratings and Fas expression, the association between pain and $\mathrm{ACTH}$, cortisol and SP levels was explored with bivariable and multivariable mixed linear models. ACTH exhibited evidence of associations with pain ratings in both bivariable $(P<0.001)$ and multivariable approaches $(P=0.05)$, whereas with regard to SP levels, a nonsignificant trend for an association with pain ratings was observed at the bivariable model only (Table 4A and B). When mixed linear models for the association between pain ratings and Fas expression were adjusted for the levels of ACTH, SP, and cortisol, simultaneously, the association of pain with Fas expression on $\mathrm{B}$ cells and cytotoxic $\mathrm{T}$ cells remained borderline significant (Table 4C); however, adjustment for 1 neuropeptide at the time did not accentuate the association between pain and Fas expression (results not shown).

\section{Discussion}

This exploratory study aimed to address for the first time the potential associations between pain and lymphocyte apoptotic susceptibility in critically ill patients through a repeated measures descriptive design. The main findings included: 1) a correlation between pain ratings and Fas expression on $\mathrm{B}$ and cytotoxic $\mathrm{T}$ cells, even after adjustment for a number of clinical factors and medications, 2) an association between pain and ACTH levels, and 3) a potential mediating effect of stress neuropeptide levels in the association between pain and Fas expression. To the degree of our knowledge, these results are novel, and they may, therefore, merit further investigation in critically ill patients and generally in individuals with pain. Moreover, the results show a considerable self-reported intensity of pain and pain indicators at rest as assessed by 2 BPSs throughout the study period in critically ill patients. Evidence originating from controlled studies showing increased infection rates and incidence of complications ${ }^{8,24}$ in critically ill patients with unrelieved pain provides support to the notion that pain suppresses immune activity in critical illness, albeit with as yet unclear mechanisms. The results suggest subset-specific lymphocyte apoptosis as a potential mechanism involved in pain-associated immunosuppression.

The role of pain in the modulation of apoptosis is not clarified adequately, especially in lymphocytes. Animal model evidence suggests that persistent pain induces neuronal apoptosis both through stress- and nonstress-mediated mechanisms. ${ }^{25}$ Moreover, experimental pain has been implicated 
Table 2 Results of linear mixed models

\begin{tabular}{|c|c|c|c|}
\hline \multicolumn{4}{|c|}{ A. Bivariable mixed models } \\
\hline Independent variable & Estimate & $95 \% \mathrm{Cl}$ & $P$-value \\
\hline \multicolumn{4}{|c|}{ Dependent variable: Fas expression on B cells } \\
\hline PAS rating & 0.243 & $0.16-0.47$ & 0.036 \\
\hline BPS rating & 1.3 & $0.43-2.18$ & 0.005 \\
\hline \multicolumn{4}{|c|}{ Dependent variable: Fas expression on cytotoxic $T$ cells } \\
\hline PAS rating & 0.29 & $0.11-0.58$ & 0.042 \\
\hline BPS rating & 0.501 & $0.29-1.29$ & 0.041 \\
\hline \multicolumn{4}{|c|}{ B. Multivariable mixed models } \\
\hline Parameter & Estimate & $95 \% \mathrm{Cl}$ & $P$-value \\
\hline \multicolumn{4}{|c|}{ Dependent variable: Fas expression on $B$ cells } \\
\hline BPS rating & 0.88 & $0.12-1.48$ & 0.052 \\
\hline MODS & -0.005 & $-0.15-0.14$ & 0.938 \\
\hline Trauma & 0.18 & $-0.52-0.89$ & 0.606 \\
\hline Opioid analgesia & 0.39 & $-0.33-1.13$ & 0.280 \\
\hline Midazolam & 0.03 & $-1.16-1.22$ & 0.958 \\
\hline Propofol & -0.25 & $-2.0 \mathrm{I}-\mathrm{I} .48$ & 0.768 \\
\hline \multicolumn{4}{|c|}{ Dependent variable: Fas expression on cytotoxic T cells } \\
\hline BPS rating & 1.035516 & $0.30-1.76$ & 0.006 \\
\hline MODS & 0.039169 & $-0.09-0.17$ & 0.556 \\
\hline Trauma & 0.488728 & $-0.09-0.17$ & 0.099 \\
\hline Opioid analgesia & 0.076353 & $-0.35-0.50$ & 0.727 \\
\hline Sedation: midazolam & 0.40 & $-0.69-1.4$ & 0.470 \\
\hline Sedation: propofol & -0.15 & $-1.4-1.1 \mid$ & 0.811 \\
\hline
\end{tabular}

Notes: (A) bivariable models for the association between pain ratings and Fas expression accounting for repeated measurements. (B) multivariable models additionally adjusting for MODS severity, the presence of trauma, and analgesia and sedation. Pain NRS self-assessments could not be included in multivariable models due to small number of ratings. For multivariable models, results taking account BPS ratings are only included. Results including PAS measurements are similar.

Abbreviations: $\mathrm{Cl}$, confidence Interval; BPS, behavioral pain scale; MODS, Multiple Organ Dysfunction Score; NRS, numeric rating scale; PAS, pain assessment scale.

in oxidative stress and apoptosis in rat hepatocytes. ${ }^{26}$ Additionally, persistent pain is involved in muscle-cell apoptosis in the affected area, despite the absence of inflammation. ${ }^{27}$

Research evidence shows an altered immune functionality in patients experiencing either acute pain, such as postoperative, ${ }^{28,29}$ or chronic pain. ${ }^{30}$ However, investigators have focused mainly on NK responses, and the effect of pain in lymphocyte apoptosis has not been addressed. The findings of increased apoptotic susceptibility in cytotoxic $\mathrm{T}$ cells are in-line with observations of decreased cytotoxic T-cell counts in patients with chronic pain..$^{30}$ A diminished Th1 response has been presumed to account for the suppressed cytotoxic T-cell proliferation. ${ }^{31}$ Furthermore, the finding that the association between pain and lymphocyte Fas expression may persist even after adjustment for disease severity, presence of trauma, and opioid analgesics and sedatives, which may exert an additional immunosuppressive effect, suggests the possibility that nociception may have a direct effect on lymphocyte survival, irrespective of diagnosis, severity, or treatment. Therefore, based on the results, it is worth exploring whether subset-specific apoptosis may also be a factor in decreased cytotoxic T-cell numbers in pain.

The observation of significant associations between $\mathrm{ACTH}$ levels and pain ratings is in line with the stress response elicited in pain. Critically ill patients experience acute pain and possibly persistent pain that may transition to chronic. ${ }^{32}$ Pain elicits a composite stress reaction promoting the release of neurotransmitters, stress peptides, and cytokines, which orchestrate an integrated neuroendocrine and immune response. ${ }^{33}$ The nonsignificant trend for an association between SP levels and pain is also worth exploring further, inasmuch as SP is involved in both pain and stress perception. This observation is in agreement with findings in acute myocardial infarction patients, where, however, associations between SP levels and pain were more accentuated. ${ }^{34}$ Moreover, the observed partial mediating effect of stress neuropeptides in the association between pain ratings and lymphocyte Fas expression is consistent with previous evidence implicating stress in the modulation of apoptosis and activation of the JNK pathway. ${ }^{35}$ Psychological stress ${ }^{36}$ and stress hormones ${ }^{37}$ have been reported to induce lymphocyte apoptosis in humans and in animal models. ${ }^{38}$ Based on the above, the role of stress peptides in the regulation of painrelated apoptosis merits to be investigated further.

The observation of increased pain ratings at rest is in accordance with previous studies in trauma and general ICU populations. ${ }^{2,39,40}$ The finding of pain at rest is essential, in view of evidence summarized earlier regarding the important

Table 3 Bivariate associations between pain ratings and Fas expression (positive cell count/subset cell count) on T and B cells

\begin{tabular}{|c|c|c|c|c|c|c|c|c|c|}
\hline \multirow[t]{2}{*}{ Pain rating } & \multicolumn{3}{|c|}{ Fas (+) B cells } & \multicolumn{3}{|c|}{ Fas (+) T helper cells } & \multicolumn{3}{|c|}{ Fas (+) cytotoxic $T$ cells } \\
\hline & First day & $\begin{array}{l}\text { Maximum } \\
\text { severity }\end{array}$ & $\begin{array}{l}\text { Minimum } \\
\text { severity }\end{array}$ & First day & $\begin{array}{l}\text { Maximum } \\
\text { severity }\end{array}$ & $\begin{array}{l}\text { Minimum } \\
\text { severity }\end{array}$ & First day & $\begin{array}{l}\text { Maximum } \\
\text { severity }\end{array}$ & $\begin{array}{l}\text { Minimum } \\
\text { severity }\end{array}$ \\
\hline $\begin{array}{l}\text { Pain NRS self- } \\
\text { assessment }\end{array}$ & 0.402 & $0.4 I I$ & $0.897^{*}$ & -0.564 & -0.216 & -0.735 & 0.355 & 0.170 & $0.832 *$ \\
\hline PAS ratings & $0.680 *$ & $0.763 * *$ & 0.122 & -0.114 & 0.178 & -0.233 & $0.448 *$ & 0.258 & 0.233 \\
\hline BPS ratings & $0.472 *$ & $0.532 * *$ & 0.063 & -0.138 & -0.118 & -0.349 & 0.343 & $0.479 *$ & 0.39 \\
\hline
\end{tabular}

Notes: Pearson's $r$ correlation coefficients are shown for the first day measurements and for the days of maximum and least severity $(n=36)$. $* P<0.05$. $* * P<0.00$ I. Abbreviations: BPS, behavioral pain scale; NRS, numeric rating scale; PAS, pain assessment scale. 
Table 4 Mixed model analysis for the association with stress neuropeptides

\begin{tabular}{|c|c|c|c|}
\hline Independent variable & Estimate & $95 \% \mathrm{Cl}$ & $P$-value \\
\hline \multicolumn{4}{|c|}{ A. Bivariable mixed models } \\
\hline \multicolumn{4}{|c|}{ Dependent variable: BPS pain ratings } \\
\hline SP & 0.59 & $0.12-0.6$ & 0.065 \\
\hline ACTH & 0.125 & $0.60-1.91$ & $<0.001$ \\
\hline Cortisol & 0.49 & $-0.03-0.12$ & 0.221 \\
\hline Parameter & Estimate & $95 \% \mathrm{Cl}$ & $\mathbf{P}$-valu \\
\hline \multicolumn{4}{|c|}{ B. Multivariable mixed models for associations with Fas expression } \\
\hline \multicolumn{4}{|c|}{ Dependent variable: Fas expression on B cells } \\
\hline SP & -0.02 & $-0.13-0.08$ & 0.626 \\
\hline ACTH & 0.01 & $-0.003-0.19$ & 0.050 \\
\hline Cortisol & 0.03 & $-0.06-0.13$ & 0.470 \\
\hline \multicolumn{4}{|c|}{ C. Dependent variable: Fas expression on cytotoxic T cells } \\
\hline \multicolumn{4}{|c|}{ a. Dependent variable: Fas expression on B cells } \\
\hline BPS rating & 0.95 & $0.45-2.16$ & 0.052 \\
\hline SP & 0.001 & $-0.43-0.43$ & 0.996 \\
\hline Cortisol & 0.14 & $-0.17-0.45$ & 0.349 \\
\hline ACTH & 0.05 & $-0.32-0.43$ & 0.765 \\
\hline \multicolumn{4}{|c|}{ b. Dependent variable: Fas expression on cytotoxic $\mathrm{T}$ cells } \\
\hline BPS rating & 0.99 & $0.12-1.91$ & 0.063 \\
\hline $\mathrm{SP}$ & -0.15 & $-0.49-0.19$ & 0.375 \\
\hline Cortisol & 0.15 & $-0.14-0.45$ & 0.291 \\
\hline ACTH & -0.02 & $-0.35-0.31$ & 0.890 \\
\hline
\end{tabular}

Notes: (A) and (B): association between stress neuropeptide levels and pain ratings (only results for BPS ratings appear; results for PAS ratings are similar). (C) Associations of pain ratings with Fas expression adjusted for stress neuropeptide levels.

Abbreviations: ACTH, adrenocorticotropic hormone; BPS, behavioral pain scale; $\mathrm{Cl}$, confidence interval; NRS, numeric rating scale; PAS, pain assessment scale; SP, substance $P$.

role of persistent pain in activating intracellular signaling cascades and apoptosis and of the effect of persistent pain in the stress response and central and peripheral sensitization.

\section{Limitations}

The results need to be interpreted with caution due to the limitations of this pilot study, mainly stemming from the small convenience sample, low power to detect significant associations and the correlational design. Convenience sampling resulted in a predominantly male study population, which reflects common demographic composition of critical care populations. ${ }^{41}$ Furthermore, the increased number of patients with neurologic injury account for an additional limitation, inasmuch as it is still unclear whether BPSs for noncommunicative patients are valid for neurological trauma patients. ${ }^{42}$ Additionally, many confounding factors are in effect due to the complexity and heterogeneity of critically ill patients, including patient history, autonomic activation status, and medication variations, which are difficult to control for in clinical research. Moreover, although only 3 stress peptides were investigated in this study, the stress response involves numerous hormones and peptides, which were not included in the present design. Despite these limitations, many of which would be difficult to overcome in a pilot study, the study highlights novel associations that merit further investigation in the future.

\section{Conclusion and implications}

These results provide preliminary evidence that pain needs to be investigated further as a factor in critical illness-related immunosuppression, through association with increased apoptotic susceptibility in B and cytotoxic T cells. Also, the results provide preliminary evidence of a potential mediating effect of stress neuropeptides in the association between pain and apoptotic susceptibility.

Important practice implications can be drawn in the future since early and systematic detection and treatment of pain, both at rest and during interventions, may contribute to the prevention of critical illness-related immunosuppression and the improvement of patients' outcomes.

\section{Acknowledgments}

This work is part of a larger study on the role of stress in critical illness, preliminary results of which have been published in Papathanassoglou ED, Mpouzika MD, Giannakopoulou M, Bozas E, Middleton N, Boti S, Karabinis A. Pilot Investigation of the Association Between Serum Stress Neuropeptide Levels and Lymphocyte Expression of Fas and Fas Ligand in Critical Illness. Biol Res Nurs 2015;17(3):285-294; Mpouzika MD, Papathanassoglou ED, Giannakopoulou M, Bozas E, Middleton N, Boti S, Patiraki EI, Karabinis A. Altered serum stress neuropeptide levels in critically ill individuals and associations with lymphocyte populations. Neuropeptides 2013;47(1):25-36. This study was supported by the Cyprus University of Technology Faculty Grant to EDEP and by the National and Kapodistrian University of Athens ELKE Grants 70/4/5688 and 70/4/6403 to EDEP and MG, respectively.

\section{Disclosure}

The authors report no conflicts of interest in this work.

\section{References}

1. Rotondi A, Chelluri L, Sirio C, et al. Patients' recollections of stressful experiences while receiving prolonged mechanical ventilation in an intensive care unit. Crit Care Med. 2002;30(4):746-752.

2. Gélinas C. Management of pain in cardiac surgery ICU patients: have we improved over time? Intensive Crit Care Nurs. 2007;23(5):298-303.

3. Puntillo K, Nelson J, Weissman D, et al. Palliative care in the ICU: relief of pain, dyspnea, and thirst - a report from the IPAL-ICU Advisory Board. Intensive Care Med. 2013;40(2):235-248.

4. Puntillo K, Max A, Timsit J, et al. Determinants of procedural pain intensity in the intensive care unit: the Europian Study. Am J Respir Crit Care Med. 2014;189(1):39-47.

5. Chanques G, Jaber S, Barbotte E, et al. Impact of systematic evaluation of pain and agitation in an intensive care unit. Crit Care Med. 2006;34(6):1691-1699. 
6. Puntillo K, Pasero C, Li D, et al. Evaluation of pain in ICU patients. Chest. 2009;135(4):1069-1074.

7. Stanik-Hutt JA, Soeken KL, Belcher AE, Fontaine DK, Gift AG. Pain experiences of traumatically injured patients in a critical care setting. Am J Crit Care. 2001;10(4):252-259.

8. Sakaue S, Sunagawa M, Tanigawa $\mathrm{H}$, et al. A single administration of morphine suppresses the reduction of the systemic immune activity caused by acute inflammatory pain in rats. Masui. 2011;60(3):336-342.

9. Sacerdote P, Bianchi M, Gaspani L, et al. The effects of tramadol and morphine on immune responses and pain after surgery in cancer patients. Anesth Analg. 2000;90(6):1411-1414.

10. Hotchkiss R, Coopersmith C, Karl I. Prevention of lymphocyte apoptosis - a potential treatment of sepsis? Clin Infect Dis. 2005;41(suppl 7): S465-S469.

11. Marshall J, Charbonney E, Gonzalez P. The immune system in critical illness. Clin Chest Med. 2008;29(4):605-616.

12. Baumann S, Krueger A, Kirchhoff S, Krammer P. Regulation of T cell apoptosis during the immune response. Curr Mol Med. 2002; 2(3):257-272.

13. Mpouzika M, Papathanassoglou E, Giannakopoulou M, et al. Altered serum stress neuropeptide levels in critically ill individuals and associations with lymphocyte populations. Neuropeptides. 2013;47(1):25-36.

14. Kaufmann T, Strasser A, Jost PJ. Fas death receptor signalling: roles of Bid and XIAP. Cell Death Differ. 2012;19(1):42-50.

15. Papathanassoglou E, Moynihan J, McDermott M, Ackerman M. Expression of Fas (CD95) and Fas ligand on peripheral blood mononuclear cells in critical illness and association with multiorgan dysfunction severity and survival. Crit Care Med. 2001;29(4):709-718.

16. Brame A, Singer M. Stressing the obvious? An allostatic look at critical illness. Crit Care Med. 2010;38(suppl 10):S600-S607.

17. Siffleet J, Young J, Nikoletti S, Shaw T. Patients' self-report of procedural pain in the intensive care unit. J Clin Nurs. 2007;16(11):2142-2148.

18. Barr J, Fraser G, Puntillo K, et al. Clinical practice guidelines for the management of pain, agitation, and delirium in adult patients in the intensive care unit. Crit Care Med. 2013;41(1):263-306.

19. Puntillo K, Miaskowski C, Kehrle K, Stannard D, Gleeson S, Nye P. Relationship between behavioral and physiological indicators of pain, critical care patients' self-reports of pain, and opioid administration. Crit Care Med. 1997;25(7):1159-1166.

20. Payen J, Bru O, Bosson J, et al. Assessing pain in critically ill sedated patients by using a behavioral pain scale. Crit Care Med. 2001; 29(12):2258-2263.

21. Goris R. Multiple-organ failure. Arch Surg. 1985;120(10):1109-1115.

22. Marshall J, Cook D, Christou N, Bernard G, Sprung C, Sibbald W. Multiple organ dysfunction score. Crit Care Med. 1995;23(10):1638-1652.

23. Knaus W, Zimmerman J, Wagner D, Draper E, Lawrence D. APACHE acute physiology and chronic health evaluation: a physiologically based classification system. Crit Care Med. 1981;9(8):591-597.

24. Payen J, Bosson J, Chanques G, Mantz J, Labarere J. Pain assessment is associated with decreased duration of mechanical ventilation in the intensive care unit. Anesthesiology. 2009;111(6):1308-1316.

25. Jalalvand E, Javan M, Haeri-Rohani A, Ahmadiani A. Stress- and nonstress-mediated mechanisms are involved in pain-induced apoptosis in hippocampus and dorsal lumbar spinal cord in rats. Neuroscience. 2008; 157(2):446-452.
26. Rezaei M, Rasekh HR, Ahmadiani A, Pourahmad J. Involvement of subcellular organelles in inflammatory pain-induced oxidative stress and apoptosis in the rat hepatocytes. Arch Iran Med. 2008;11(4):407-417.

27. Gradl G, Gaida S, Gierer P, Mittlmeier T, Vollmar B. In vivo evidence for apoptosis, but not inflammation in the hindlimb muscle of neuropathic rats. Pain. 2004;112(1):121-130.

28. Beilin B, Shavit Y, Trabekin E, et al. The effects of postoperative pain management on immune response to surgery. Anesth Analg. 2003; 97(3):822-827.

29. Yermal S, Witek-Janusek L, Peterson J, Mathews H. Perioperative pain, psychological distress, and immune function in men undergoing prostatectomy for cancer of the prostate. Biol Res Nurs. 2009;11(4): 351-362.

30. Vines S, Gupta S, Whiteside T, Dostal-Johnson D, Hummler-Davis A. The relationship between chronic pain, immune function, depression, and health behaviors. Biol Res Nurs. 2003;5(1):18-29.

31. Kaufmann I, Eisner C, Richter P, et al. Lymphocyte subsets and the role of TH1/TH2 balance in stressed chronic pain patients. Neuroimmunomodulation. 2007;14(5):272-280.

32. Papathanassoglou E. Recent advances in understanding pain: what lies ahead for critical care? Nurs Crit Care. 2014;19(3):110-113.

33. Chapman C, Tuckett R, Song C. Pain and stress in a systems perspective: reciprocal neural, endocrine, and immune interactions. J Pain. 2008; 9(2):122-145.

34. Kletsiou E, Giannakopoulou M, Bozas E, Iliodromitis E, AnastasiouNana M, Papathanasoglou E. Chest pain due to coronary artery disease alters stress neuropeptide levels: potential implications for clinical assessment. Eur J Cardiovasc Nurs. 2009;8:S128.

35. Shen CP, Tsimberg Y, Salvadore C, Meller E. Activation of Erk and JNK MAPK pathways by acute swim stress in rat brain regions. $B M C$ Neurosci. 2004;5:36.

36. Sakami S, Nakata A, Yamamura T, Kawamura N. Psychological stress increases human $\mathrm{T}$ cell apoptosis in vitro. Neuroimmunomodulation. 2002;10(4):224-231.

37. Lucin K, Sanders V, Popovich P. Stress hormones collaborate to induce lymphocyte apoptosis after high level spinal cord injury. J Neurochem. 2009;110(5):1409-1421.

38. Yin D, Tuthill D, Mufson R, Shi Y. Chronic restraint stress promotes lymphocyte apoptosis by modulating CD95 expression. $J$ Exp Med. 2000;191(8):1423-1428.

39. Chanques G, Sebbane M, Barbotte E, Viel E, Eledjam J, Jaber S. A prospective study of pain at rest: incidence and characteristics of an unrecognized symptom in surgical and trauma versus medical intensive care unit patients. Anesthesiology. 2007;107(5):858-860.

40. Deutschman CS, Ahrens T, Cairns CB, Sessler CN, Parsons PE. Critical Care Societies Collaborative/USCIITG Task Force on Critical Care Research: multisociety task force for critical care research: key issues and recommendations. Chest. 2012;141(1):201-209.

41. Papathanassoglou E, Middleton N, Hegadoren K. Sex-dependent disparities in critical illness: methodological implications for critical care research. Nurs Crit Care. 2015;20(2):58-62.

42. Arbour C, Gélinas C, Loiselle C, Bourgault P. An exploratory study of the bilateral bispectral index for pain detection in traumatic-braininjured patients with altered level of consciousness. J Neurosci Nurs. 2015;47(3):166-177.
Journal of Pain Research

\section{Publish your work in this journal}

The Journal of Pain Research is an international, peer reviewed, open access, online journal that welcomes laboratory and clinical findings in the fields of pain research and the prevention and management of pain. Original research, reviews, symposium reports, hypothesis formation and commentaries are all considered for publication.
The manuscript management system is completely online and includes a very quick and fair peer-review system, which is all easy to use. Visit http://www.dovepress.com/testimonials.php to read real quotes from published authors. 